\title{
LAS POSIBILIDADES DE INTEGRACIÓN DEL TRANSPORTE ESCOLAR Y REGULAR EN ARAGÓN
}

\author{
R. Badía Lázaro \\ Departamento de Geografía y Ordenación del Territorio \\ Universidad de Zaragoza \\ C/ Pedro Cerbuna 12, 50009 Zaragoza \\ rbadia@unizar.es
}

\begin{abstract}
Resumen: El presente artículo explora las ventajas y el potencial de integrar las líneas de transporte escolar en la red de transporte regular de viajeros en Aragón. Con este propósito, se ha recopilado información sobre los puntos de origen, destino y características básicas de todas las rutas escolares. Los resultados muestran la incipiente aparición de dos modelos de integración diferentes. El primero de ellos se corresponde con las rutas escolares integradas cuya principal ventaja es la mejora en la accesibilidad de la población rural a los servicios. El segundo son las rutas regulares con reserva de plaza cuyos beneficios son un menor coste para la administración y una mejora de la rentabilidad de las líneas regulares. Ambos modelos todavía cubren una pequeña parte de la demanda por lo que atendiendo a sus ventajas tienen un gran potencial de expansión.
\end{abstract}

Palabras clave: accesibilidad, centro educativo, transporte escolar, transporte rural, red de transporte.

\begin{abstract}
This paper explores the advantages and the potential of integrating the school transport routes in the public transport network in the region of Aragón (Spain). For this propose I have compiled information about the departure points, destination points and the basic characteristics of all the routes. The results show the incipient appearance of two different integrated models. The first one corresponds to the integrated route whose main advantage is the improvement in the accessibility of services for the rural population. The second model is public transport routes with
\end{abstract}

Recibido: 10-12-2016. Aceptado: 20-12-2016. 
booked seats in which the main benefits are their lower cost and an improvement in the profit of public transport companies. These models still cover a small part of the demand so taking into account their advantages they have a great potential for expansion.

Keywords: accessibility, educational center, school transport, rural transport, transport network.

\section{Introducción y objetivos}

El transporte escolar se perfila como un instrumento de vital importancia en núcleos de población de pequeño tamaño demográfico que no disponen de colegio o instituto dado que permite el traslado de los alumnos desde sus domicilios hasta el centro educativo de referencia. Este tipo de transporte asegura que los estudiantes de medios rurales puedan ejercer en igualdad de condiciones su derecho a la educación. De forma paralela el transporte público en áreas rurales juega un importante papel al permitir a los colectivos no motorizados el acceso a aquellos servicios, en muchas ocasiones de carácter básico, de los que carecen en sus núcleos de origen. Con la finalidad de mejorar las oportunidades de viaje de este último colectivo se vienen aplicando medidas de integración del transporte regular y escolar.

Los estudios que se centran en la integración de ambos tipos de transporte son escasos. En el ámbito europeo destaca el proyecto Actions on the integration of Rural Transport Services ARTS (2003), financiado por el programa marco de Investigación y Desarrollo Tecnológico de la Dirección General de Movilidad y Energía de la Comisión Europea. El objetivo era probar diferentes acciones para mejorar el transporte en zonas rurales centrándose en la integración del transporte escolar y regular en 3 de las 8 demostraciones. El principal resultado fue la mejora de la accesibilidad de la población rural a los centros de servicios cercanos (Transport research and innovation portal, a.). Otro ejemplo es el proyecto "Byabussen" en el que se crearon rutas en integración planificadas de forma que sirvieran como alimentador al servicio de transporte público en una región rural del sur de Suecia (Transport research and innovation portal, b.).

Actualmente en Europa las acciones de integración del transporte escolar y regular suelen formar parte de planes más ambiciosos de mejora del transporte rural que en muchas ocasiones traen consigo la implantación de sistemas de transporte a la demanda (DRT). Reino Unido, Francia y Alemania cuentan con un mayor número de ejemplos de estos sistemas (Oña, 2010), de entre ellos sólo unos pocos, como los británicos Wigglybus o PhoneandGo (Brake 2004), integran flujos de viajeros escolares y regulares. Estudios recientes abordan la creación de Zonas de Transporte Únicas (TTAs) en el Reino Unido. Dichas zonas suponen un nuevo paso en la integración al incorporar también rutas de transporte por motivos sanitarios o sociales (Raikes, 2015). 
En España existe un menor número de estudios al respecto. El primer gran hito se identifica con la realización de una demostración del citado proyecto ARTS en un área rural de la provincia de Orense. En este caso concreto, "se mejoraba así la reducida oferta de los servicios de transporte convencionales y a la par se incrementaban los beneficios de los operadores" (ARTS, 2003: 94-110 en Delgado, 2016: 97). Sin embargo, el proyecto de mayor envergadura se corresponde con la puesta en servicio de un sistema de transporte a la demanda en amplias zonas rurales de Castilla y León. Este sistema integró las primeras rutas escolares, en parte, como medida paliativa a la supresión de itinerarios de transporte llevadas a cabo por las políticas de recorte derivadas de la crisis económica (Delgado, 2016).

En el caso de Aragón la autoridad competente también ha optado por la integración del transporte escolar y regular intentando compensar la problemática del sector en ámbitos rurales. Esta región posee una densidad de población de 27,6 hab/ $\mathrm{km}^{2}$ (Instituto Aragonés de Estadística, 2015), con 14 comarcas por debajo del umbral de $10 \mathrm{hab} / \mathrm{km}^{2}$. Además es un territorio fuertemente envejecido y con una gran dispersión del poblamiento. En cuanto a los problemas referentes al transporte podemos citar una demanda débil y dispersa, la existencia de plazas libres, tiempos de recorrido relativamente largos, coste elevado para la administración o la segregación de flujos. Este último se traduce en la existencia de dos redes de transporte diferentes y paralelas en el territorio; la red de transporte público y la red de transporte escolar. A su vez, dentro de la red de transporte escolar encontramos dos redes, una para movilizar a alumnos de primaria y otra para transportar a estudiantes de secundaria.

Con la intención de mejorar el transporte rural el gobierno de Aragón ha comenzado a integrar las rutas escolares con las líneas de tráfico regular. La finalidad de esta política es acabar con la segregación de flujos de tráfico en áreas con una demanda débil y dispersa. Para ello se están aplicando dos nuevos tipos de modelos. El primero son las rutas en reserva de plaza, en el que los escolares viajan en líneas de transporte público regular. El segundo son las rutas integradas, que se corresponden con rutas escolares que admiten a usuarios de transporte público. Además, dentro de las rutas escolares también se han creado nuevas rutas mixtas, que integran a alumnos de primaria y secundaria.

Este trabajo estudia, por primera vez, la situación de la red de transporte escolar en Aragón tras la incorporación de estos nuevos modelos. Por tanto, el objetivo general de este estudio es:

- Explorar las ventajas y el potencial de expansión de las líneas de transporte escolar con modelos de integración en la red de transporte regular de viajeros en Aragón.

Para poder dar respuesta a este objetivo general se plantean otros dos objetivos específicos que son: 
1. Identificar los puntos de origen y de destino del transporte escolar.

2. Determinar las características y distribución de las líneas de transporte escolar con modelos de integración.

A lo largo del trabajo se pone de manifiesto la buena cobertura de la red de transporte escolar en el territorio, con la existencia de una gran cantidad de puntos de origen y de destino, lo cual denota un fuerte potencial de integración. Además se determina que de los dos nuevos modelos implantados, uno presenta efectos positivos al generar un aumento de oportunidades de viaje de la población rural, mientras que otro disminuye el coste del servicio. En consecuencia, se identifican los ámbitos más adecuados para implantar ambos modelos de la forma más ventajosa.

La estructura de este artículo se organiza de la siguiente manera. Tras esta introducción, se tratan los aspectos normativos del transporte escolar en Aragón. A continuación se expone la metodología de estudio. El cuarto capítulo hace referencia a los resultados de la investigación, que se discuten en el apartado siguiente. A partir de lo expuesto en esta discusión se extraen las principales conclusiones.

\section{Características básicas del transporte escolar en Aragón}

En el curso 2013-2014 se encuentran matriculados en Aragón un total de 209.046 alumnos. (Instituto Aragonés de Estadística, a, b). La distribución de los alumnos en el territorio aragonés presenta, conjuntamente con la distribución de la población y otros muchos indicadores socioeconómicos, fuertes contrastes. Por un lado, la delimitación comarcal de Zaragoza cuenta con el 60\% de los alumnos de Aragón (124.000). Sin embargo, la tónica general es la existencia de comarcas donde el número de alumnos es bajo, de hecho, 9 de ellas poseen menos de 1.000 alumnos (Instituto Aragonés de la Juventud, 2014). En estas últimas la escasez de población genera una fuerte presión sobre la red de transporte escolar a la hora de conectar la débil demanda con los centros educativos.

El transporte escolar en Aragón queda regulado por una serie de normas que condicionan de forma directa sus características. En primer lugar, la ley orgánica 2/2006, de 3 de mayo, de Educación (Boletín Oficial del Estado, 2006), establece en su artículo 82.2 la obligatoriedad de un servicio de transporte escolar gratuito que traslade aquellos alumnos que no disponen de servicios educativos en su municipio a otro cercano donde si los haya. Los pliegos de prescripciones técnicas (Gobierno de Aragón, 2014, a, b y c.) determinan que el tiempo de trayecto de un alumno no puede superar los 60 minutos por viaje. La orden de 14 de mayo de 2013, de la Consejera de Educación, Universidad, Cultura y Deporte, dicta las normas para la organización y funcionamiento del servicio complementario de transporte escolar en la Comunidad Autónoma de Aragón. En su artículo 1.2 determina dos modalidades de transporte escolar: 
a) Rutas de transporte escolar establecidas mediante contratos, convenios y reserva de plazas en líneas regulares y que tienen por destino centros de enseñanza públicos de niveles educativos no universitarios.

b) Ayudas individualizadas de transporte escolar para los casos en que no exista ruta de transporte organizado. Estas ayudas se materializan en acuerdos entre padres de escolares que suelen llevar por su cuenta a uno o varios de estos alumnos a sus centros de estudios en vehículo privado.

Las rutas de transporte escolar movilizan a más de 14.000 estudiantes en Aragón, mientras que las ayudas individualizadas sólo benefician a 900 alumnos según datos de la dirección general de ordenación académica de la consejería de educación de la DGA. Por ello, el objeto de este estudio se centra únicamente en los desplazamientos realizados mediante estas rutas.

El artículo 3.1 de la citada orden determina junto con el artículo 10 del Decreto 24/2008 del 12 de febrero, del Gobierno de Aragón las diferentes modalidades del transporte escolar en Aragón. Dichas modalidades son las siguientes:

a) La contratación del servicio a empresas del sector para uso exclusivo de escolares. Esta modalidad es la que denominaremos en el trabajo como rutas escolares estándar o convencionales.

b) La integración de dichas líneas escolares estándar en el transporte regular de viajeros en zonas de débil tráfico. Esto supone "la utilización de la capacidad residual de los vehículos de más de nueve plazas que presten servicios de transporte escolar (...), para la admisión de usuarios demandantes de transporte público general' (Art. 10 del Decreto 24/2008 del 12 de febrero, del Gobierno de Aragón).

c) La contratación del servicio de transporte escolar a través de la reserva de plazas en líneas de transporte público regular existentes.

d) Además la prestación del servicio de transporte escolar en cualquiera de las tres modalidades previamente descritas se puede realizar mediante el establecimiento de convenios de colaboración con Corporaciones y Entidades Locales, como ocurre en los casos de las cuatro comarcas pirenaicas y las Cuencas Mineras.

En resumen, por un lado, tenemos la existencia de tres modelos de transporte escolar diferenciados: las rutas escolares estándar, las integradas y las de reserva de plaza, siendo estos dos últimos modelos integrados de transporte. Por ello, durante el trabajo al mencionar las rutas integradas nos referiremos al segundo modelo, mientras que al hablar de modelos integrados estaremos incluyendo a estas y también a las rutas con reserva de plaza. Por otro lado, nos encontramos con dos órganos gestores diferentes, que son, el departamento de ordenación académica del gobierno de Aragón y los convenios con entidades locales. Bajo estos convenios se opera el transporte escolar de las cuatro comarcas pirenaicas, las Cuencas Mineras y el municipio de Barbastro en cualquiera de las tres modalidades expuestas con anterioridad. 


\section{Metodología}

\subsection{Fuentes de información}

Para poder dar respuesta a los objetivos de este estudio se requiere información de dos temáticas diferentes, la red de transporte escolar y los centros escolares. Las fuentes utilizadas para ello son las siguientes:

- La principal fuente estadística en este proyecto la representan los datos de rutas de transporte escolar. Se trata de un conjunto de datos inéditos en formato Excel que han sido cedidos por la consejería de educación de la DGA. Estos configuran la única fuente oficial y completa de rutas escolares en Aragón. En ellos se recoge un listado con las principales características de las rutas. Su explotación presentó problemas en el trabajo debido a que cada uno de los registros se corresponde con una ruta, mientras que para nuestro análisis es necesario conocer la información desagregada por núcleos. Esta información estaba contenida en un campo de tipo texto que describe el itinerario y el número de alumnos recogidos en cada lugar, sin embargo, no aparecen desagregados por nivel educativo. Otro problema destacable fue la utilización de códigos de ruta no concordantes entre direcciones provinciales por lo que hubo que crear un código de itinerario propio. En general, se trata de una fuente fiable pero cuya explotación a escala de núcleos es dificultosa.

Para obtener la información de centros educativos se han manejado las siguientes fuentes.

- Datos de oferta educativa disponibles en la Carpeta escolar de Aragón 20132014 publicada por el Instituto Aragonés de la Juventud. En este documento se detallan todos los centros existentes en cada núcleo de población, así como, su titularidad y los niveles educativos a los que atienden. Se trata por tanto de información valiosa para el trabajo, sin embargo, estos datos vienen en formato pdf por lo que deben de ser digitalizados.

- Datos de centros que reciben transporte escolar cedida por la Dirección General de Ordenación Académica de la consejería de educación de la DGA. Esta fuente nos permite conocer los destinos de todas las rutas de transporte escolar ya que contiene, entre otros datos, el nombre del centro y el municipio de destino. De nuevo se trata de información en formato pdf lo que dificulta su tratamiento.

Además de las fuentes de información hasta ahora descritas, que son fuentes de tipo estadístico, se ha recurrido a una serie de fuentes cartográficas para la realización de los mapas. Las fuentes cartográficas utilizadas en este trabajo se han descargado de la Infraestructura de Datos Espaciales del Gobierno de Aragón y están referidas al sistema 
de coordenadas oficial ETRS 89. Las capas utilizadas son las entidades de población, los municipios y las comarcas de Aragón. La elección de colores en la cartografía se ha adaptado a lo aconsejado en el sitio web Colour Advise for Maps. (Brewer, C. Harrower, M).

\subsection{Tratamiento de la información}

Una vez obtenidos los datos tanto de centros como de rutas escolares se ha procedido a su tratamiento, explotación y análisis de resultados. A partir de estos datos se han construido tres tablas diferentes, orígenes, destinos y rutas de transporte escolar.

La tabla de destinos recoge datos de las dos fuentes utilizadas para conocer la oferta educativa de Aragón. En esta tabla cada uno de los registros hace referencia a cada uno de los centros educativos que reciben transporte escolar. Los atributos de estos registros, nivel educativo y núcleo de población principalmente se obtienen de la Carpeta escolar de Aragón (Instituto Aragonés de la Juventud, 2014). Posteriormente se asocian los núcleos de población con su código INE para su posterior representación en el SIG.

En segundo lugar se crea la tabla de rutas unificando la información procedente de seis hojas de cálculo cedidas por la consejería de educación de la DGA y que proceden de diferentes organismos. Después se añadieron los siguientes campos: un código de itinerario, códigos INE de las localidades de origen y destino, el nivel educativo, el tipo de ruta, y el organismo gestor. La Tabla 1 ofrece una descripción tanto de los campos existentes como de los añadidos, así como su fuente para estos últimos.

Una vez generada la tabla resultante se explotó de dos maneras. En primer lugar, dio lugar a los mapas de tipos de rutas de transporte escolar. Para la creación de estos se realizó un estudio piloto de digitalización de rutas. Sin embargo, la gran extensión de la red junto al hecho de que muchas rutas circulan en paralelo desaconsejó su representación lineal. Como consecuencia, finalmente esta tipología se presentó sobre los núcleos de población utilizando la variable visual color. Esta solución permite representar sin problemas la tipología de rutas de cada núcleo en detrimento de la estructura de la red, que queda desdibujada especialmente en las áreas más densas.

En segundo lugar, esta tabla también es explotada en el apartado 4.2.3 de caracterización de la red de transporte escolar. Mediante la funcionalidad de tablas dinámicas de Microsoft Excel calculamos el número medio de viajeros por ruta, el número total de viajeros (multiplicando para ello el número de alumnos por los 175 días lectivos del curso 2013-2014), la media de kilómetros recorridos, el coste por kilómetro y el coste por viajero. Todos estos datos son analizados en el citado apartado.

La tercera, y última, tabla creada es la tabla de demanda que contiene datos de la demanda escolar desagregada por núcleos y niveles educativos. Para generar esta ta- 
Tabla 1. Campos de la base de datos de rutas

\begin{tabular}{|c|c|c|}
\hline & Campo & Descripción \\
\hline \multirow{9}{*}{ 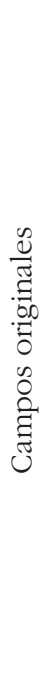 } & Código DGA & Código de ruta asignado por la DGA. \\
\hline & Origen & Denominación de la localidad de origen. \\
\hline & Descripción & $\begin{array}{l}\text { Campo de texto con la descripción de los núcleos que conforman } \\
\text { el recorrido de la ruta con referencia al número de alumnos que } \\
\text { acceden en cada núcleo entre paréntesis. }\end{array}$ \\
\hline & Destino & Nombre del centro de destino. \\
\hline & Alumnos según nivel & $\begin{array}{l}\text { Cinco campos que contienen el número de alumnos de educación } \\
\text { especial, infantil, primaria, secundaria, y postobligatoria de cada ruta. }\end{array}$ \\
\hline & Horarios & $\begin{array}{l}\text { Cuatro campos similares que recogen los horarios de salida y lle- } \\
\text { gada tanto de mañana como de tarde. }\end{array}$ \\
\hline & Kilómetros & El número de kilómetros recorridos al día. \\
\hline & Precio & Precio diario. \\
\hline & Observaciones & $\begin{array}{l}\text { Descripción de cualquier característica especial de la ruta, si la hu- } \\
\text { biere. }\end{array}$ \\
\hline \multirow{6}{*}{ 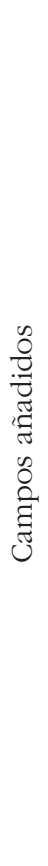 } & Código Itinerario & $\begin{array}{l}\text { Identificador único para cada uno de los itinerarios de una mis- } \\
\text { ma ruta. Contiene el número de provincia, el número de ruta pro- } \\
\text { cedente del código DGA y se añade el número de itinerario. } \\
\text { Ejemplo: Z101I2. Se construye ante la existencia de itinerarios con } \\
\text { el mismo código en el campo código DGA. }\end{array}$ \\
\hline & Código Origen & \multirow{2}{*}{$\begin{array}{l}\text { Código INE del núcleo de origen/destino. Se obtiene mediante } \\
\text { el procedimiento explicado en la tabla de destinos. }\end{array}$} \\
\hline & Código Destino & \\
\hline & Nivel educativo & $\begin{array}{l}\text { Describe el nivel educativo de la ruta, primaria, secundaria, edu- } \\
\text { cación especial y ruta mixta, mediante las letras "P", "S", "E" y "M" } \\
\text { respectivamente. Se extrae a partir de la información de alumnos } \\
\text { desagregada por niveles que contiene la misma tabla }\end{array}$ \\
\hline & Tipo de ruta & $\begin{array}{l}\text { Especifica el modelo de transporte escolar, ruta estándar, inte- } \\
\text { grada o en reserva de plazas, mediante las letras "E" "I" y "R" res- } \\
\text { pectivamente. Su fuente es el código de ruta de la DGA que con- } \\
\text { tiene iniciales alusivas a este hecho. }\end{array}$ \\
\hline & Organismo & $\begin{array}{l}\text { Recoge el organismo gestor de las rutas, que puede ser directamente } \\
\text { la DGA o mediante convenio, se expresa mediante las letras "D" y } \\
\text { "C" respectivamente. Esta información se deriva de la organización } \\
\text { en diferentes hojas Excel previa a la unificación de la tabla. }\end{array}$ \\
\hline
\end{tabular}


bla se ha utilizado la información contenida en el campo descripción (dónde aparecen los núcleos que conforman el recorrido de la ruta y el número de alumnos recogidos). Tomando como referente este campo se ha tenido que replicar cada una de las rutas tantas veces como núcleos existan en la descripción de su itinerario. En cada nuevo registro se han cambiado los campos con el nombre y código del núcleo de origen por los de cada uno de los núcleos en los que para una determinada ruta. El campo de descripción no indica el nivel educativo de los alumnos, sin embargo, conocemos si la ruta es de primaria, secundaria o mixta. Por ello se han creado tres campos (uno para el total de alumnos de infantil y primaria, otro para los alumnos de educación secundaria y postobligatoria y otro para alumnos de cualquier nivel) que se rellenan en función del nivel educativo de la ruta. De este modo se ha podido separar la demanda a excepción de las rutas mixtas (que representa sólo 16 rutas de 529). Con esta información se ha creado una cartografía de demanda para primaria y otra para secundaria. En ambas cartografías aparecen las rutas mixtas.

\section{Resultados}

\subsection{Orígenes y destinos del transporte escolar}

El primer objetivo específico planteado en este trabajo consiste en identificar los puntos de origen y de destino del transporte escolar. La finalidad es conocer la magnitud y la cobertura del transporte escolar en el territorio ya que este es un factor clave a la hora de determinar el impacto que podría tener su integración. Para conocer los puntos de origen vamos a utilizar la demanda de transporte escolar, mientras que, para conocer los lugares de destino vamos a utilizar la oferta de transporte escolar.

\subsubsection{Núcleos de origen}

La demanda total de transporte escolar en Aragón está compuesta por 14.454 alumnos, de estos 2.104 son de primaria, 11.417 de secundaria y 933 de educación especial (Dirección general de ordenación académica, 2014, a.). Esto supone que un 78\% de dicha demanda se corresponde con estudiantes de secundaria algo que se explica por la menor frecuencia espacial de los institutos en el territorio. La dominancia del nivel educativo de secundaria va a quedar patente en muchos otros parámetros del transporte escolar que se irán exponiendo en este trabajo.

El Mapa 1 recoge la distribución de los puntos de origen de todos los alumnos que utilizan el servicio de transporte escolar en Aragón, sea cual sea su nivel educativo. El dimensionado de las esferas representa la cantidad de alumnos demandantes de trans- 


\section{DEMANDA DE TRANSPORTE ESCOLAR EN ARAGÓN: CURSO 2013-2014}

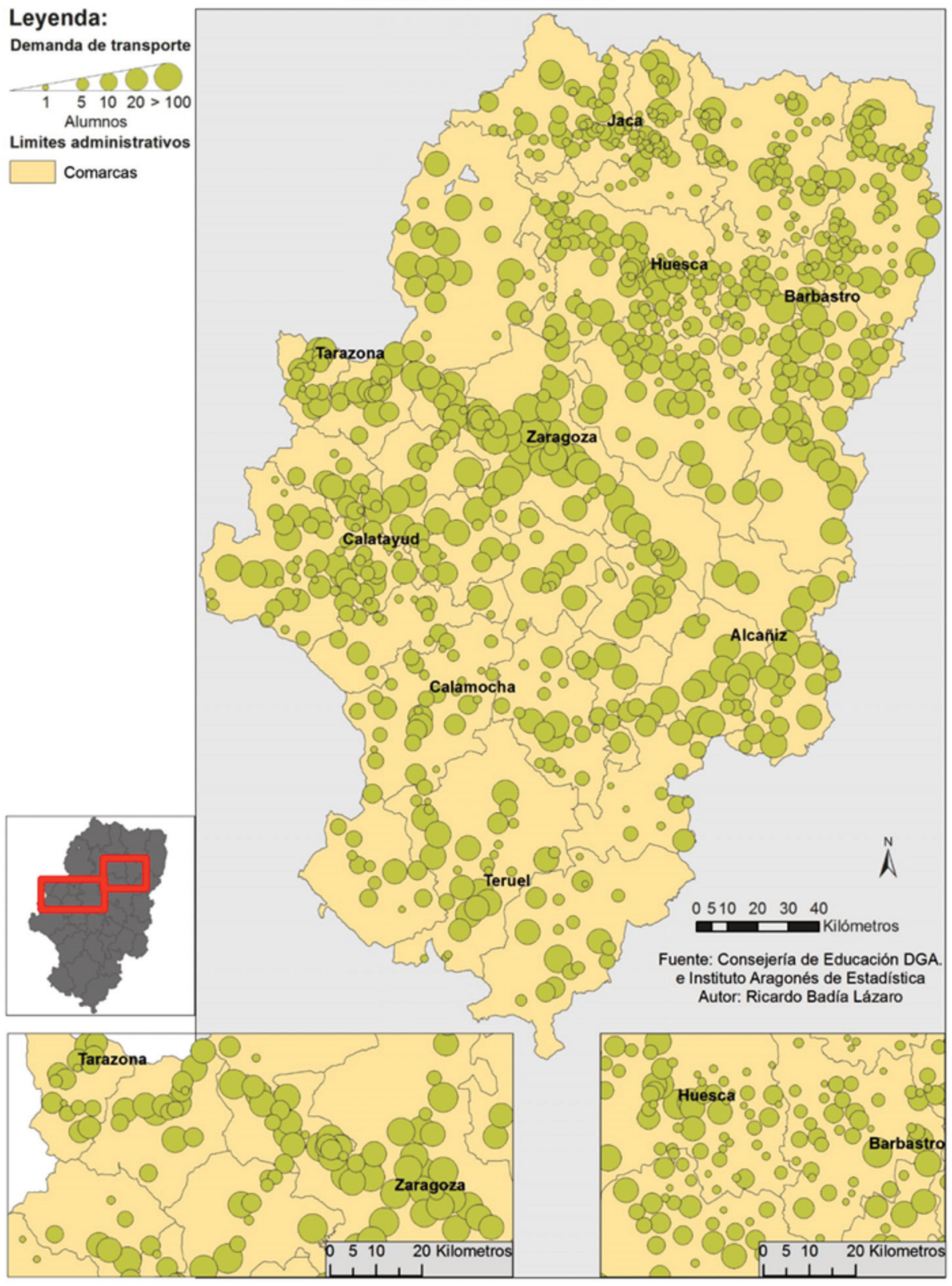

Mapa 1. Demanda de transporte escolar en Aragón (2014). 
porte en cada núcleo. (Para conocer la situación concreta de la demanda en educación primaria y secundaria véanse Mapa 3 y Mapa 4 respectivamente).

En términos generales podemos observar cómo la demanda de transporte escolar, que totaliza 889 puntos de origen, se distribuye por todo el territorio aragonés. Esta se encuentra dispersa por todas las áreas rurales de la región ya que debido a la naturaleza de este servicio la demanda se focaliza en los núcleos más pequeños, al ser estos los que presentan una menor oferta educativa.

La distribución de los puntos de origen está muy relacionada con las características del sistema de asentamientos. De esta manera existen áreas donde los núcleos se concentran en ejes como es el caso del Valle del Ebro, Jalón, Jiloca, Huecha etc... Mientras que en otras zonas, entre las que se encuentran los somontanos, las comarcas pirenaicas o zonas de montaña de la provincia de Teruel, la dispersión de núcleos es mayor. Esta dispersión dificulta la prestación del servicio de transporte escolar.

Sin embargo, el mayor condicionante de este servicio es el número de alumnos a transportar en cada núcleo. La demanda es mayor en el área central de la depresión del Ebro y en el área oeste de la provincia de Huesca. Por el contrario, ésta es menor en las zonas montañosas de las provincias de Huesca y en gran parte de la provincia de Teruel. Esto significa que de nuevo en estas zonas la prestación del servicio de transporte escolar será más costosa.

\subsubsection{Núcleos de destino}

Tras conocer los núcleos de origen centraremos el análisis en conocer la oferta educativa de la comunidad, ya que estos centros se corresponden con los destinos de las líneas de transporte escolar. Los centros educativos siguen una jerarquía en el territorio que es ampliamente conocida. Los colegios son más numerosos y se sitúan en núcleos de escaso tamaño demográfico, mientras que los institutos se localizan en municipios de mayor tamaño. Como hemos visto, la demanda de transporte escolar en Aragón es esencialmente de educación secundaria por lo que podemos afirmar que la mayoría de los destinos de los alumnos se encuentran en núcleos de población de cierto tamaño.

El Mapa 2 muestra la distribución de los centros educativos de primaria y secundaria que cuentan con transporte escolar en Aragón. El primer hecho destacable en este mapa es que el número de puntos de destino es sensiblemente inferior al de orígenes. A pesar de eso constituyen un número importante de lugares, que se distribuyen de forma equitativa por todo el territorio aragonés. Su localización tiene una gran importancia de cara a las posibilidades de integración de las rutas, cómo se verá en el apartado de discusión de este trabajo, siendo preferibles los núcleos de mayor tamaño demográfico o localidades situadas en importantes ejes de comunicaciones. 


\section{CENTROS CON OFERTA DE TRANSPORTE ESCOLAR EN ARAGÓN: CURSO 2013-2014}

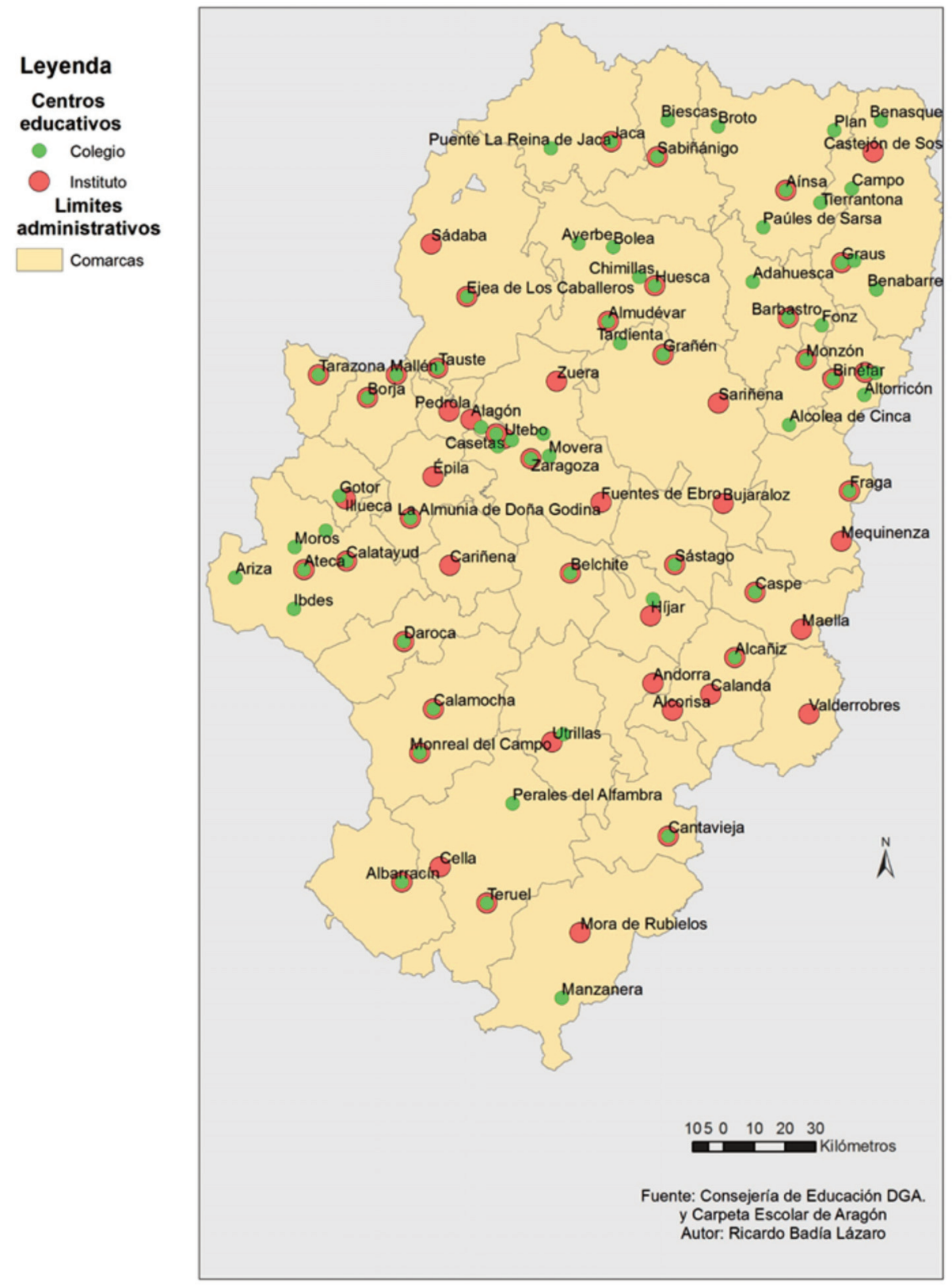

Mapa 2. Centros con oferta de transporte escolar en Aragón (2014). 


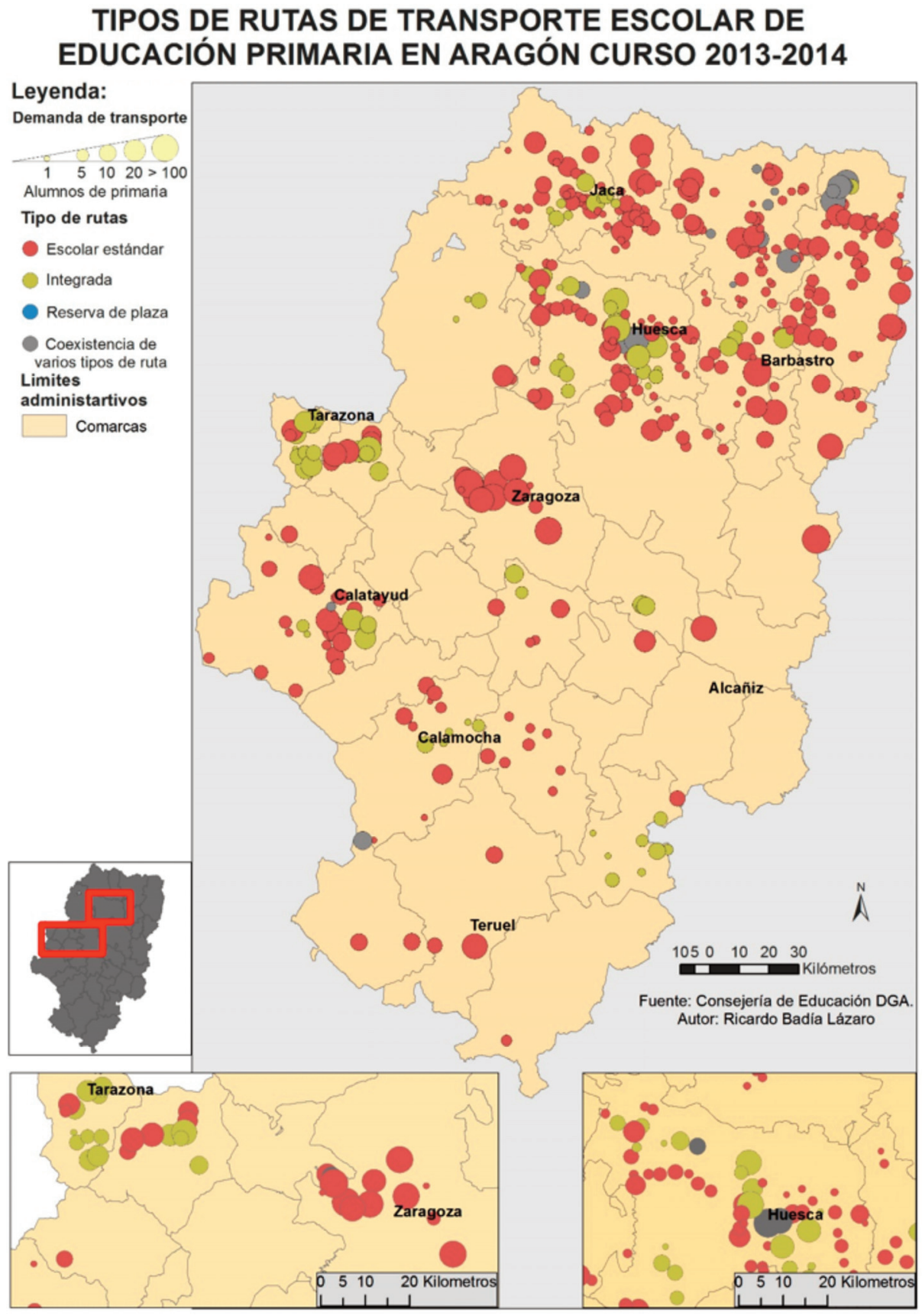

Mapa 3. Tipos de rutas de transporte escolar de educación primaria en Aragón (2014). 


\section{TIPOS DE RUTAS DE TRANSPORTE ESCOLAR DE}

\section{EDUCACIÓN SECUNDARIA EN ARAGÓN CURSO 2013-2014}

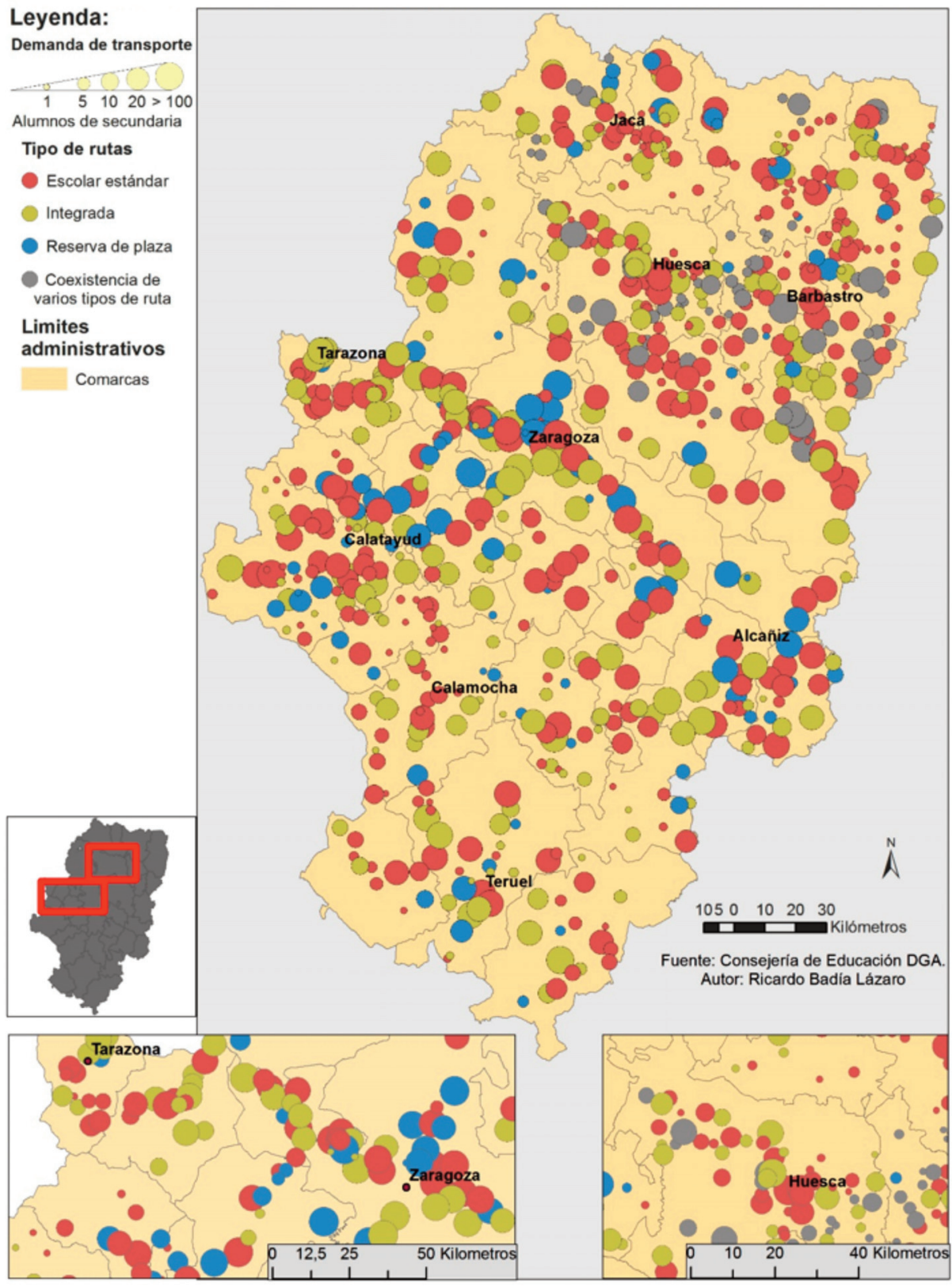

Mapa 4. Tipos de rutas de transporte escolar de educación secundaria en Aragón (2014). 
Los destinos de educación primaria, prestan mayor atención a las zonas rurales, de hecho, muchos de ellos se encuentran en núcleos de escasa población y quedan desconectados de ejes de transporte (Ibdes, Adahuesca, etc...). Sin embargo, también encontramos localidades insertas en ejes de transporte (Manzanera en el eje Zaragoza-Valencia o Puente la Reina de Jaca en el eje Huesca-Pamplona), además de núcleos de cierto tamaño demográfico que suelen ser también receptores de alumnos de secundaria. Por lo tanto, las localidades de destino para los estudiantes de secundaria son, por lo general, núcleos de mayor entidad, de hecho, muchos de ellos son cabeceras comarcales.

\subsection{Análisis de la red de transportes}

Tras determinar los núcleos de origen y destino de los estudiantes pasamos a analizar la red que permite hacer de nexo de unión entre estos puntos. Su análisis permitirá dar respuesta al segundo de los objetivos específicos del trabajo que busca determinar las características y distribución de las líneas de transporte escolar con modelos de integración. A partir de su lectura estaremos en disposición de alcanzar el objetivo general del trabajo que consiste en explorar las ventajas y el potencial de expansión de las líneas de transporte escolar integradas en la red de transporte regular de viajeros en Aragón.

\subsubsection{Tipología de rutas}

La comunidad autónoma de Aragón contaba para el curso 2013-2014 con 529 rutas de transporte escolar. (Dirección general de ordenación académica, 2014, a). Este estudio las clasifica conforme a dos tipologías diferentes que quedan sintetizadas en la Tabla 2.

La primera categorización hace referencia al nivel educativo de los alumnos transportados. Las rutas de secundaria son las más utilizadas con un 60\% del total (321 rutas). Recordemos que estas atienden al grueso de la demanda. Otro hecho a destacar es la existencia de rutas mixtas que trasladan en un mismo viaje a estudiantes de primaria y de secundaria. Estas rutas cuentan con una proporción testimonial puesto que por razones operativas no pueden extenderse sistemáticamente debido a la disparidad de destinos entre ambos niveles educativos.

La segunda tipología clasifica las rutas en tres categorías: rutas estándar o convencionales, rutas integradas y rutas con reserva de plaza. Como se ha mencionado en el apartado 2 las rutas integradas se corresponden con rutas escolares donde se permite el acceso a cualquier usuario del transporte público, mientras que, aquellas con reserva de plaza son rutas de transporte público regular en las que se reserva un cierto número de asientos a los estudiantes. La Tabla 2 muestra que las rutas estándar, es decir, las que todavía no presentan ningún modelo de integración contabilizan 370 (un 70\% del 
Tabla 2. Número de rutas según tipología

\begin{tabular}{|l|c|c|c|c|}
\hline Rutas & \multicolumn{3}{|c|}{ Según tipo de ruta } & \multirow{2}{*}{ Total } \\
\cline { 1 - 4 } Según nivel educativo & Estándar & Integrada & Reserva & \\
\hline Primaria & 112 & 22 & & 134 \\
Secundaria & 188 & 81 & 52 & 321 \\
Especial & 55 & 3 & & 58 \\
Mixto & 15 & 1 & & 16 \\
\hline Total & 370 & 107 & 52 & 529 \\
\hline
\end{tabular}

Fuente: Dirección general de ordenación académica. Consejería de Educación DGA. Elaboración propia.

total). La segunda tipología más común se corresponde con las líneas integradas que suman 107 (un 20\%). La tipología menos representada es la de rutas en reserva de plaza que suponen un 10\%. Cabe destacar que educación secundaria es el nivel educativo con una mayor proporción de líneas con modelos de integración al poseer 81 de las 107 rutas integradas y la totalidad de las 52 rutas en reserva de plaza.

\subsubsection{Distribución de las rutas por el territorio}

Una vez analizadas las tipologías de ruta existentes en Aragón, vamos a conocer la distribución espacial de las rutas de transporte escolar en función de su tipología. En primer lugar, hay que destacar que la segmentación de la demanda y oferta de transporte escolar según niveles educativos se resuelve en la existencia de dos redes diferentes en el territorio. La primera red, es la red de educación primaria, que sirve para transportar a los alumnos de educación infantil y primaria hasta los núcleos con colegio. La segunda red, es la red de secundaria que hace lo propio con los alumnos de E.S.O. y bachillerato con destino en los institutos.

El Mapa 3 y el Mapa 4 representan las redes de primaria y secundaria respectivamente. Estos simbolizan el modelo de ruta (estándar, integrada o en reserva de plazas) mediante implantación puntual sobre cada uno de los núcleos de población con demanda de transporte escolar utilizando para ello la variable visual color. El tamaño de las esferas de cada municipio representa la propia demanda de transporte escolar.

A primera vista llama la atención que los municipios pertenecientes a la red de transporte escolar de educación secundaria cubren todo el territorio aragonés. Sin embargo, la red de educación primaria deja partes importantes sin cubrir. La principal causa de este vacío es la existencia de amplias áreas (Centro de la depresión del Ebro, Bajo Aragón) dónde todos los municipios tienen colegios. En el resto de áreas la causa es la 
débil demanda, que se cubre con ayudas individuales de transporte escolar y que por lo tanto, no dan lugar a rutas.

Centrando la atención en la red de educación primaria expuesta en el Mapa 3 se evidencia el dominio de las rutas estándar. En cuanto a las rutas integradas, estas presentan un número modesto y siguen un patrón de ubicación aleatorio en el territorio, con la existencia de comarcas como Hoya de Huesca o Tarazona y el Moncayo donde su proporción es mayor.

La red de educación secundaria (Mapa 4) posee los tres modelos de transporte escolar comentados, incluyendo las rutas en reserva de plaza, que no se han implantado todavía en educación primaria. Estos tres modelos coexisten en prácticamente todo el territorio aragonés aunque un análisis pormenorizado resalta algunas pequeñas diferencias. Estas diferencias en su distribución están relacionadas principalmente con la propia naturaleza de las rutas. De esta manera, las rutas en reserva de plaza se encuentran en áreas dónde el servicio de transporte público es mejor. Así gran parte de esta tipología se asienta sobre los principales corredores de transporte de Aragón (Valles del Ebro y del Jalón y ejes de Madrid, Valencia y Castellón), o en el entorno de la capital. En consecuencia encontramos una mayor importancia de estas rutas en la provincia de Zaragoza. Por contra, las rutas integradas se encuentran situadas fuera de los principales ejes viarios de la comunidad, donde la red de transporte público presenta un nivel de servicio menor y la posibilidad de integración mediante reserva de plazas disminuye. Estas rutas cubren generalmente a localidades más pequeñas y están más representadas en las provincias de Huesca y Teruel.

Un resultado inesperado ha sido que en ambos mapas la mayoría de núcleos con varios destinos (municipios en los que, por ejemplo, los alumnos de secundaria viajan a un instituto, mientras que los de bachillerato continúan hasta otro más lejano) se concentran en la provincia de Huesca. Esto se debe a la existencia de muchos institutos en los que sólo se imparte la E.S.O. en esta provincia (Instituto Aragonés de la juventud, 2014) pero también a que las diferentes direcciones provinciales tratan el transporte escolar de diferente forma.

\subsubsection{Características de las rutas}

Al igual que ocurría con la distribución, las características de las rutas varían en función de su naturaleza. A continuación vamos a examinar diversos parámetros relacionados con el uso de las mismas, su longitud y su coste económico según tipologías. Para conocer estas características contamos con los datos de rutas de transporte escolar que han sido cedidos por la consejería de educación de la DGA. Estos datos contemplan el número de alumnos, la longitud y el coste de las rutas. A partir de ellos, obtenemos el número total de alumnos transportados en un año, el coste diario por alumno y el coste por kilómetro recorrido. 
La Tabla 3 y la Tabla 4 resumen estas cifras para educación primaria y educación secundaria respectivamente. El número total de alumnos transportados fue mayor en secundaria donde se realizaron 3,2 millones de desplazamientos. Esta cifra contrasta fuertemente con los 0,6 millones del transporte de primaria, volviendo a poner de manifiesto la importancia del transporte de educación secundaria sobre el total.

Tabla 3. Parámetros principales de las rutas de transporte escolar en educación primaria (2014)

\begin{tabular}{|l|c|c|c|c|}
\hline Rutas de primaria & \multicolumn{3}{|c|}{ Según tipo de ruta } & \multirow{2}{*}{ Total } \\
\hline Características & Estándar & Integrada & Reserva & \\
\hline $\begin{array}{l}\text { Número de alumnos } \\
\text { transportados por viaje }\end{array}$ & 16,1 & 21,7 & - & 17,2 \\
\hline $\begin{array}{l}\text { Número de viajes } \\
\text { realizados curso 2013-2014 }\end{array}$ & 485.501 & 129.593 & - & 615.094 \\
\hline Longitud media en km & 41,5 & 44,8 & - & 42,1 \\
\hline Coste diario por alumno & 8,09 & 8,22 & - & 8,13 \\
\hline Coste por kilómetro & 1,57 & 2,14 & - & 1,67 \\
\hline
\end{tabular}

Fuente: Dirección general de ordenación académica. Consejería de Educación DGA. Elaboración propia.

Tabla 4. Parámetros principales de las rutas de transporte escolar en educación secundaria (2014)

\begin{tabular}{|l|c|c|c|c|}
\hline Rutas de secundaria & \multicolumn{3}{|c|}{ Según tipo de ruta } & \multirow{2}{*}{ Total } \\
\cline { 1 - 3 } Características & Estándar & Integrada & Reserva & \\
\hline $\begin{array}{l}\text { Número de alumnos } \\
\text { transportados por viaje }\end{array}$ & 37,8 & 31,6 & 37,4 & 36,2 \\
\hline $\begin{array}{l}\text { Número de viajes } \\
\text { realizados curso 2013-2014 }\end{array}$ & 1.930 .594 & 694.427 & 498.542 & 3.123 .563 \\
\hline Longitud media en km & 45,3 & 57,2 & 43,8 & 48,1 \\
\hline Coste diario por alumno & 4,46 & 6,80 & 3,41 & 4,80 \\
\hline Coste por kilómetro & 1,89 & 1,93 & 1,38 & 1,83 \\
\hline
\end{tabular}

Fuente: Dirección general de ordenación académica. Consejería de Educación DGA. Elaboración propia. 
De las tres modalidades de transporte escolar comentados la ruta estándar fue el modelo más utilizado contabilizando casi medio millón de viajes de alumnos de primaria y prácticamente 2 millones para los de secundaria (Ver Tabla 3 y Tabla 4). El resto de parámetros se aproxima a los valores medios, en parte, por su mayor peso sobre el total. Destaca el hecho de que el coste diario por alumno en educación primaria es casi el doble que en secundaria $(8,09 €$ frente a $4,46 €)$ a pesar de que el coste por kilómetro es similar. Este desfase se explica por una menor ocupación de los vehículos en el transporte de primaria y la ausencia de rutas en reserva de plazas. En términos generales el coste de las rutas estándar presenta valores intermedios entre los otros dos modelos existentes.

Las rutas integradas son el segundo modelo de transporte más utilizado, contabilizando a 134.000 estudiantes de primaria y más de 700.000 en educación secundaria, valores que se sitúan significativamente por debajo de las rutas estándar. Estas rutas se caracterizan por ser las más caras tanto en educación primaria como en secundaria. Sin embargo, es en este último nivel educativo en el que encontramos el mayor desfase ya que el coste por alumno de las rutas integradas es $6,80 €$, lo que supone $2 €$ más que la media $(4,80 €)$.

Las rutas en reserva de plaza son las menos utilizadas con un total de 515.000 viajes realizados exclusivamente en educación secundaria. Sus características se asemejan a las rutas estándar aunque sus costes son bastante inferiores $(3,41 €$ por kilómetro y $1,38 €$ por alumno). Por lo tanto, la reserva de plaza se configura como el modelo más económico ya que aplican la tarifa de las líneas regulares.

Los resultados de este capítulo indican la existencia de dos redes diferenciadas, una para transportar a alumnos de primaria, y otra para transportar a alumnos de secundaria. Existen tres modelos de transporte diferentes. De estos modelos, el más extendido es la ruta estándar que presenta unos costes intermedios. El segundo modelo más utilizado es el modelo de integración que es el que posee unos costes más elevados. Por último, el modelo en reserva de plazas se configura como el más barato y como el menos extendido en el territorio. Estos resultados por lo tanto nos llevan al capítulo de discusión en el que se tienen en cuenta estos datos así como la incidencia en el número de oportunidades de viaje para determinar qué tipo de modelo debe de aplicarse en función de las características territoriales.

\section{Discusión}

Los resultados obtenidos a lo largo del trabajo permiten describir la situación del transporte escolar en Aragón. Este se perfila como un sistema extenso, con una gran cantidad de puntos de origen y de destino, articulado por un número importante de líneas, y que cubre de forma equitativa a todo el territorio. Se trata de un sistema segmentado en dos subredes, una que atiende a educación primaria, y otra a secundaria, 
aunque existen algunas rutas mixtas. Sin embargo, la característica más importante es que se trata de un sistema que todavía presenta un bajo nivel de integración con el transporte regular de viajeros, especialmente en educación primaria. El sistema cuenta con dos modelos de integración radicalmente distintos y que aparecen de forma incipiente en el territorio. Por lo tanto, respondiendo al objetivo general planteado en el trabajo podemos decir que existe un gran potencial para extender los modelos de integración y beneficiarse de sus diferentes ventajas. A continuación vamos a discutir las principales ventajas y desventajas de cada uno de los modelos de integración y en función de estas vamos a atribuir cada uno de los modelos a territorios con características distintas. Esto permitirá proponer una nueva organización para el transporte escolar en Aragón más beneficiosa para escolares y usuarios del transporte público.

En primer lugar, vamos a tratar sobre las ventajas e inconvenientes de las rutas integradas. Estas se configuran como el modelo de integración más utilizado en Aragón. El primer hecho a destacar es que por su naturaleza, este modelo representa una nueva oportunidad de viaje para la población al permitir el ascenso y descenso de viajeros en una ruta que previamente era exclusiva del transporte escolar. Sin embargo, en Aragón se ha evidenciado como el modelo más caro, con un coste por alumno superior al de las rutas estándar, a pesar de que se nutre de un ingreso adicional, la venta de billetes a los usuarios del transporte público. La causa que explica este mayor coste es que este modelo se ha empezado a implantar en aquellas rutas más deficitarias, de hecho el Mapa 4 mostró como las líneas en integración circulan por zonas con una ruralidad marcada, precisamente como medida para combatir este déficit. De cualquier modo, se trata de un modelo más caro que el de reserva de plazas.

El modelo en reserva de plaza es el menos utilizado en la actualidad. Su principal ventaja es el aprovechamiento de los servicios de transporte público preexistentes para movilizar a los alumnos sin necesidad de una ruta escolar y sin perjuicio para los mismos. Esto permite incurrir en unos costes por alumno muy inferiores. Sin embargo, no suponen una mejora para los usuarios del transporte público. Además se trata de un modelo más difícil de implantar en educación primaria, dónde los alumnos deberían movilizarse con un acompañante en todo momento (Boletín Oficial de Aragón, 2013).

Además de los modelos de transporte escolar, otro aspecto a discutir es la existencia de rutas mixtas en dónde los alumnos de primaria y secundaria viajan juntos. En Aragón existen 16 rutas de este tipo principalmente centradas en áreas de marcado carácter rural. Las ventajas de estas rutas son el aumento de la ocupación y un menor coste para la administración. Sin embargo, presentan muchas restricciones para su implantación, por la inadecuación de horarios y de lugares de destino entre ambos niveles educativos. Esto supone una importante restricción a su extensión territorial.

Teniendo en cuenta las ventajas e inconvenientes de los modelos de transporte escolar se puede aventurar, a grandes rasgos, la configuración de un nuevo mapa de transporte escolar en Aragón. 
En esta nueva situación el modelo de transporte escolar en integración sería el dominante ya que es el que mayores ventajas presenta para las áreas rurales profundas, muy presentes en esta Comunidad Autónoma. Este hecho se debe a que es en estos ámbitos dónde se aprovecha más su principal ventaja, la creación de una nueva oportunidad de viaje para la población. Los resultados muestran que los orígenes de las rutas escolares se sitúan principalmente en localidades de escaso tamaño demográfico. Por ello, la integración masiva de estas rutas permitiría la creación de nuevas oportunidades de viaje en estos pequeños núcleos, que son por lo general, dónde el sistema de transporte público presenta unas menores prestaciones. En cambio, los destinos de las líneas se ubican en núcleos con cierto tamaño demográfico. De este modo, la integración permitiría la mejora de la accesibilidad de la población rural a los centros de servicios más cercanos, y no sólo eso, también a paradas con un servicio de transporte público mejor. Esto supone una gran ventaja ya que con una reordenación de horarios que facilitase los trasbordos entre las líneas integradas y las regulares se podrían extender las oportunidades de viaje hasta ciudades de mayor envergadura.

Las rutas integradas no son el modelo con el menor coste económico pero la venta de billetes a usuarios del transporte regular reduciría su coste, aunque fuera de forma ligera. Además hay que recordar la obligatoriedad que la administración tiene de facilitar transporte escolar a los alumnos que lo necesiten independientemente del coste económico derivado de su ubicación según lo establecido en la ley orgánica 2/2006, de 3 de mayo, de Educación (Boletín Oficial del Estado, 2006). Esto justifica el mantenimiento de estas rutas por lo que se su integración permitiría disfrutar de un menor coste y mayores ventajas para el resto de la población.

La utilización del modelo de reserva de plazas en este nuevo mapa de transporte escolar sería más limitada que en el caso anterior, pero superaría los niveles actuales. Estas rutas quedarían asociadas a áreas que poseen un buen sistema de transporte público, como pueden ser los municipios integrantes del Consorcio de Transportes del área de Zaragoza, o localidades situadas en ejes de comunicaciones de importancia regional. En estos lugares ya existen suficientes oportunidades de viaje por lo que se propone la prestación del servicio de transporte escolar mediante la reserva de plazas incurriendo así en un menor coste.

El crecimiento de los dos modelos anteriores se haría a costa de las rutas integradas estándar cuya utilización sería mínima, quedando relegadas únicamente a casos especiales o líneas en las que no se disponga de espacio restante suficiente para poder acomodar a todos los estudiantes. Se recomienda la aplicación del modelo en reserva de plazas también para los estudiantes de educación primaria que viajarían en las líneas regulares con acompañante conforme a la legislación vigente (Boletín Oficial de Aragón, 2013).

En lo referente a las rutas mixtas estas seguirían jugando un papel marginal. Debido a su naturaleza estas rutas sólo son eficaces en aquellos lugares en los que los desti- 
nos de educación primaria y educación secundaria coinciden y cuando los horarios son similares. Por lo tanto, la implantación de estas rutas requiere un esfuerzo adicional de coordinación de horarios entre colegios e institutos de la zona para poder acomodar a los alumnos de diferentes niveles en el máximo número de viajes posible. Este esfuerzo adicional sólo compensa en comarcas con una fuerte ruralidad dónde el tráfico de estudiantes es muy débil. De hecho, por el momento todas las rutas mixtas en Aragón siguen este patrón.

En resumen, se propone para Aragón avanzar hacia un sistema de transporte escolar dominado por dos modelos diferentes. El modelo más extendido en el territorio sería el de rutas escolares integradas que supondrían un aumento de las oportunidades de viaje para la población rural. En segundo lugar, las rutas en reserva de plaza se aplicarían en aquellos lugares con un buen sistema de transporte público, permitiendo reducir así el coste para la administración a la par que mejoraría los resultados de explotación de las empresas concesionarias de las rutas regulares por el sustancial incremento de viajeros.

\section{Conclusiones}

La escasez de población supone un gran problema para la prestación de servicios de transporte en el medio rural aragonés por la existencia de una demanda débil y dispersa. Dicha demanda debe atenderse de forma global sea cual sea su motivo y no mediante redes segmentadas. Por ello, la reciente implantación de dos nuevos modelos de transporte escolar integrado en Aragón (en concreto, rutas en integración y rutas en reserva de plaza) ha traído consigo claros beneficios al acabar con esta fragmentación. Por tanto se propone ir hacia un sistema de transporte escolar dominado por diferentes modelos de integración en función de las características territoriales de cada zona.

De este modo, el modelo de rutas integradas se torna como el más adecuado en áreas con una ruralidad marcada caracterizadas por un transporte público escaso o incluso inexistente. Esto es así porque permite mejorar el acceso de la población no motorizada a los núcleos de servicios cercanos. De esta forma la ruta de transporte además de garantizar el derecho de los estudiantes rurales a una educación en igualdad de condiciones, también garantiza al resto de la población el acceso a los servicios básicos. El segundo modelo a implantar se corresponde con las rutas en reserva de plaza al ser el de menor coste para la administración a la par que mejoraría los resultados de explotación de las empresas concesionarias de las rutas regulares por el sustancial incremento de viajeros. En este caso, se recomienda su implantación sólo cuando el sistema de transporte público proporcione suficientes oportunidades de viaje en las franjas horarias del transporte escolar. 
La extensión de estos modelos no está exenta de retos. En primer lugar, las rutas escolares deben de presentar horarios y paradas coordinados con las líneas regulares para que puedan funcionar como alimentadoras de las mismas mejorando la eficiencia del sistema. Otra dificultad es hacer frente a una red dinámica pues aunque los destinos y horarios no suelen cambiar de un curso para otro los itinerarios y las paradas si lo hacen. Además este servicio desaparece durante el periodo estival por lo que habrá que buscar soluciones que sigan garantizando la accesibilidad de la población rural.

En definitiva, la integración escolar ayuda a mitigar los problemas de desplazamiento de los colectivos sin acceso al vehículo privado en ámbitos rurales y por tanto es deseable para el caso de Aragón. Sin embargo, esta problemática puede ser abordada desde una perspectiva más global mediante la utilización conjunta de sistemas de transporte a la demanda e integración escolar, algo que sin duda ayudaría a hacer frente al dinamismo y la estacionalidad del transporte escolar.

\section{Agradecimientos}

En primer lugar, me gustaría agradecer al departamento de Ordenación Académica de la DGA la cesión de datos inéditos sin los cuales la realización de esta investigación no hubiera sido posible. También quiero dar las gracias al profesor D. Enrique Ruiz Budría del departamento de Geografía y Ordenación del Territorio de la Universidad de Zaragoza por todo el apoyo prestado durante las fases de investigación y tratamiento de la información que fructificaron en este artículo. Por último, deseo expresar mi más sincero agradecimiento a D. Eugenio Climent López, profesor del citado departamento, por su ayuda, consejos y sugerencias durante la fase de redacción del presente artículo.

\section{Bibliografía}

Boletín Oficial de Aragón, 2013. ORDEN de 14 de mayo de 2013, de la Consejera de Educación, Universidad, Cultura y Deporte, por la que se dictan normas para la organización y funcionamiento del servicio complementario de transporte escolar en la Comunidad Autónoma de Aragón. 113, pp. 13302-13306.

Boletín Oficial del Estado, 2006. Ley Orgánica 2/2006, de 3 de mayo, de Educación. Jefatura del Estado. BOE-A-2006-7899, pp. 17158-17207.

Brake, J., Nelson, J.D. y Wright, S. 2004. Demand responsive transport: towards the emergence of a new market segment. Journal of Transport Geography 12, pp. 323-337.

Brewer, C. y Harrower, M. Color Advise for maps. Disponible en: Colorbrewer2.org (Consultado el 03.12.2016). 
Delgado, J.M. y Martínez, L.C. 2016. El transporte a la demanda como sistema de movilidad alternativa en áreas rurales de baja densidad demográfica. El caso de Castilla y León. Boletín de la Asociación de Geógrafos Españoles 72, pp. 195-220.

Dirección general de ordenación académica. Consejería de Educación. Diputación General de Aragón, 2014, a. Datos de rutas escolares en Aragón. Zaragoza: Inédito.

Dirección general de ordenación académica. Consejería de Educación. Diputación General de Aragón, 2014, b. Relación de centros que reciben transporte escolar. Inédito.

Gobierno de Aragón, 2014, a. Pliego de prescripciones técnicas por las que se han de regir los contratos de transporte escolar. Servicio provincial de educación universidad cultura y deporte. Huesca.

Gobierno de Aragón, 2014, b. Pliego de prescripciones técnicas por las que se han de regir los contratos de transporte escolar. Servicio Provincial de Educación Universidad Cultura y Deporte. Teruel.

Gobierno de Aragón, 2014, c. Pliego de prescripciones técnicas por las que se han de regir los contratos de transporte escolar. Servicio Provincial de Educación Universidad Cultura y Deporte. Zaragoza.

Instituto Aragonés de Estadística, a. Alumnos matriculados en enseñanzas de Régimen General por comarcas. Disponible en: http://www.aragon.es/DepartamentosOrganismosPublicos/Institutos/InstitutoAragonesEstadistica/AreasTematicas/03_Educacion_Y_Formacion/01_Ense\%C3 \%B1anza_NO_Universitaria/ci.03_Ense\%C3\%B1anza_Regimen_General.detalleDepartamento? channelSelected $=0$ (Consultado el 07.12.2016).

Instituto Aragonés de Estadística, b. Centros de enseñanza de Régimen General por comarcas. Disponible en: Instituto Aragonés de Estadística. Disponible en: http://www.aragon.es/DepartamentosOrganismosPublicos/Organismos/InstitutoAragonesEstadist ica/AreasTematicas/03_Educacion_Y_Formacion/01_Enseñanza_NO_Universitaria/ci.03_Enseñan-za_Regimen_General.detalleDepartamento?channelSelected=0 (Consultado el 07.12.2016).

Instituto Aragonés de Estadística, 2015. Datos básicos de Aragón. Zaragoza.

Instituto Aragonés de la Juventud, 2014. Estudiar en Aragón: Oferta educativa Curso 2013- 2014. La carpeta escolar de Aragón. 160, pp. 6-38.

Oña, J. 2010. Una revisión de más de 50 experiencias de transporte a la demanda en Europa. I Foro internacional de transporte rural: Transporte a la demanda Valladolid, 21 de abril de 2010.

Raikes, L., Straw, W., Linton, C., 2015. Total Transport Authorities. A new deal for town and rural bus services. Londres: Institute for Public Policy Research.

Transport research and innovation portal, a. Actions on the integration of rural transport services. Disponible en: rural-transport.net (Consultado el 20.11.16).

Transport research and innovation portal, b. Integrated rural public transport - part one: Evaluation of "Byabussen" in Ystad. Disponible en: www.transport-research.info/web/projects/ project_details.cfm?ID=7969 (Consultado el 21.11.2016). 